\title{
Are randomized controlled trials sufficient evidence to guide clinical practice in Type II (non-insulin-dependent) diabetes mellitus?
}

\author{
S. Vijan ${ }^{1}$, D.M. Kent ${ }^{2}$, R.A.Hayward ${ }^{1}$ \\ ${ }^{1}$ The Veterans Affairs Center for Practice Management and Outcomes Research, Ann Arbor, Michigan, USA \\ ${ }^{2}$ The Robert Wood Johnson Clinical Scholars Program, University of Michigan, USA
}

\section{Abstract}

Randomized controlled trials (RCTs) are often considered the standard for defining the practice of evidence-based medicine. Taken alone, they are, however, often insufficient to guide clinical care. Randomized controlled trials are clearly the best method to determine whether interventions are efficacious. They have, however, numerous limitations which make them difficult to carry out or limit applicability to routine clinical practice. Although observational studies also have inherent limitations, they provide data which can help to further explain the results of randomized controlled trials. The use of observational studies to frame randomized trials can allow better application of randomized controlled trial results to individual patients and can thus help to optimize delivery of care, inform clinical practice and determine the need for further such trials.
Contrary to the claims of some enthusiasts of evidence-based medicine, randomized clinical trials often provide insufficient evidence to guide clinical care. Some proponents of "evidence-based" medicine argue that only randomized clinical trials (RCTs) should be used to define treatment recommendations, as potential confounders and biases inherent in observational studies severely limit the strength of their conclusions [1]. Although such arguments can be extreme, they highlight the belief that data from non-randomized studies are second-rate and that the results should be discounted. We argue that whereas RCTs are clearly the appropriate gold standard for establishing potentially causal associations, they often have substantial limitations in guiding clinical practice.

Observational and experimental methods both have important and complementary roles in defining

Corresponding author: S. Vijan, MD, VA HSR\&D, P.O. Box 130170, Ann Arbor, MI 48113-0170, USA

Abbreviations: RCTs, Randomized controlled trials; NNT, number needed to treat; PORT, Patient and Outcomes Research Team. optimal care, in setting evidence-based guidelines and informing sound health policy [2-12].

As a leading and increasingly important determinant of health care costs and adverse outcomes, Type II (non-insulin-dependent) diabetes mellitus is of particular importance [13]. The discussion of the United Kingdom Prospective Diabetes Study (UKPDS), a large RCT of stepped intensive therapy for nearly 4000 patients with newly diagnosed Type II diabetes, presents an excellent opportunity to review several issues in the interpretation and application of clinical research when evaluating evidence and deciding on treatments for individual patients [5, 14-17]. Commonly cited conclusions of the UKPDS include that reducing glycosylated haemoglobin improves patient outcomes and that medical intervention to achieve near-normal glycaemic control is important unless a patient has a very limited life expectancy [18]. Unfortunately, as we will discuss, this conclusion is difficult to justify using only the results of the UKPDS. In addition, we will highlight some of the difficulties of application of randomized trials to clinical practice and the use of methods which could help to overcome some of these limitations. 


\section{Randomized trials establish causality}

Randomized, controlled clinical trials are the standard for defining causality [19]. Observational studies, which can suggest causal relations, rarely are taken as definitive proof [19]. For example, although many observational studies in diabetes suggested that hyperglycaemia was closely related to the risk of developing microvascular and neuropathic complications of diabetes $[20,21]$, there remained the possibility that the analyses did not account for potential confounders. Thus, RCTs such as the DCCT were needed to show that treatment of hyperglycaemia could delay the onset and progression of early microvascular disease in Type I (insulin dependent) diabetes mellitus [4]. Whereas the UKPDS [14, 15] confirmed the relation, observational data strongly suggested that the causal relation established in the DCCT could be extrapolated to Type II diabetes. Indeed, all of the observational data supported a similar relation $[20,21]$, and simulation models predicted the microvascular outcomes of the UKPDS before the completion of the study [6-8]. In the case of the UKPDS, the study had been planned before the results of many observational studies and the DCCT; in addition, vitally important issues besides associations between microvascular disease and glycaemic control (e.g., the effect of blood pressure control on microvascular and macrovascular outcomes, previous concerning results about sulphonylureas and macrovascular risk and the potential benefit of different drug classes on macrovascular outcomes) were being addressed and it was therefore clearly appropriate to continue the study. We argue, however, that once a causal relation has been established by a large, wellconducted RCT, the scarce resources for funding RCTs should be targeted mainly at examining areas where observational data suggest that there could be heterogeneity in a causal association. For example, if observational data showed that the relation between hyperglycaemia and risk of early microvascular disease was substantially different in Type I and Type II diabetes, then a separate study in Type II diabetes would have been a high priority. Given the available observational data it was, however, striking that very few believed that another RCT was necessary to establish a causal link between hyperglycaemia and intermediate microvascular outcomes in Type II diabetes.

Nonetheless, no matter how strongly observational studies suggest a relation between exposure and outcome, this must not be confused with accepting that a causal link exists in the absence of experimental data. There are situations where treatments based on observational studies could cause harm; an excellent example is beta-carotene as preventive therapy for lung cancer. Despite strong observational data suggesting benefit, RCTs suggest that beta-carotene supplements may actually cause harm [22]. There are currently a number of recommended treatments for patients with diabetes, including, for example, primary prevention of coronary disease by treatment of hypertriglyceridaemia [23], for which randomized data supporting therapy do not exist. In situations such as these, experimental evidence is essential to establish a causal link between treatment and outcome.

\section{Randomized trials are often insufficient to guide clinical practice}

Although RCTs are considered the gold standard for establishing treatment efficacy and causality, it is impossible for RCTs to answer all clinically relevant questions. There are both fundamental limits inherent in the design of RCTs and practical limits to the information we can get from RCTs.

Fundamental limits of RCTs. By definition, RCTs are conducted under "experimental" circumstances $[10$, $19,24,25]$. The artificial nature of the experimental conditions often limit our ability to extrapolate the results of RCTs directly into clinical practice [26]. Randomized controlled trials are usually driven by protocol and involve intense interventions and follow-up that cannot be replicated in routine practice. In addition, many patients are simply unwilling to undergo random selection. Those who do volunteer possibly do not have similar baseline risks and risk reduction as those who do not volunteer [24, 25], especially when subjects are recruited from subspecialty practices or referral centres or when we would expect the intervention's effectiveness and safety to vary with patients' motivation and capabilities [27]. Thus, because the participants in RCTs are unlikely to be representative of the general population, we often cannot interpret experimental results in a direct and decisive manner despite the ideological purity of conducting a randomized trial.

Additionally, trials pool the experience of many different people to provide statistical descriptions of average outcomes. These results can be misinterpreted when presented as relative risk of outcomes. Thus, advocates of evidence-based medicine suggest that the use of statistics such as number needed to treat (NNT) and absolute risk reduction be used to help determine the true impact of an intervention [28, 29]. In the UKPDS, for example, the relative risk of any diabetes-related end point with the intensive glycaemic control policy is 0.88 , a statistically significant result ( $p=0.029)$; this has led many to suggest that all patients with Type II diabetes, unless facing limited life expectancy, should have optimal glycaemic control as a treatment goal [18]. The absolute risk reduction (per 1000 patient years) associated with treat- 
ment is, however, $5.1 \%$ ( $40.9 \%$ vs $46.0 \%)$, which equates to a NNT of 20 . In other words, 20 patients will need to be intensively controlled in order to avoid a single diabetes-related end point. This gives a much clearer picture of the magnitude of the effect and could lead to a different conclusion than simply noting a statistically significant reduction in event rates. Statistics such as NNT frame treatment efficacy in more understandable terms for both clinicians and patients.

Unfortunately, statistics based on mean results do not help clinicians apply the results of RCTs to individual patients. There are a number of limits when attempting to use RCTs to make treatment decisions. For example, RCTs that purposely recruit a relatively homogeneous cohort (such as a cardiovascular trial that includes mainly Caucasian, high-risk men) possibly do not apply to an individual patient. It has been shown that when the treatments are simple and the condition well defined, results usually can be extrapolated to the general population, as efficacy is often consistent across subgroups [11]. Unfortunately, in Type II diabetes, the disease is heterogeneous and treatments complex and extrapolation from studies of seemingly homogeneous groups to the general population must be made with caution. Studies of heterogeneous cohorts, in contrast, also present difficulty when extrapolating to individual patients, as the results of an RCT on a heterogeneous cohort are possibly driven by a small sub-group and do not apply to all patients equally [30].

Tools to evaluate the applicability of RCTs to individual patients have been proposed. Sub-group analysis is one method of dealing with such issues but such analyses are difficult to conduct well, cannot answer all relevant questions, and can lead to sample size requirements that make studies difficult [31-35]. Thus, we suggest that the more formal analyses described below, which use observational data to frame the results of RCTs, could allow better application of clinical trial results to individual patients.

Practical limits of RCTs. Randomized trials are expensive and time consuming and are often incapable of evaluating many clinically important end points due to either rare or long-term outcomes. In Type II diabetes, for example, the relative rarity of end-stage microvascular outcomes and the time course of the disease have thus far prevented RCTs from finding statistically or clinically significant differences in end-stage outcomes $[14,15]$. Thus, making treatment recommendations based solely on the experimental results could lead to clinically absurd decisions. For example, if we propose that only experimental data be considered when practicing "evidence-based" medicine, then we must realize that with a 6.2 year median follow-up in the Diabetes Control and Complications Study and 10-year follow-up in the
UKPDS, it was not possible to find any improvement in patient function (e.g., decreased blindness, amputation or renal failure), quality of life or survival [4, 14]. Should we therefore conclude that tight control will not benefit any patients? It is only through extrapolating from the intermediate outcomes of these clinical trials (e.g., incidence of early retinopathy and nephropathy) using combined results of RCTs and observational studies that we find that almost all patients with Type I diabetes and those with early onset of Type II diabetes would be expected to receive benefit from intensive glycaemic control [6-8]. Therefore, unless one is willing to accept the use of observational data and simulations to frame the results of RCTs, a strong argument for tight control in these patients cannot be made.

Randomized controlled trials also become increasingly difficult as we develop more complex treatment options. Linear growth in treatment options leads to an exponential increase in the number of potential treatment combinations that need to be evaluated; for example, evaluating 2 available treatments requires two study arms but 5 treatments could be combined in as many as 120 study arms [36]. The UKPDS is again an apt example. The study has been criticized as being complex and difficult to interpret, yet it did not evaluate many potential combinations of therapy to lower glucose in Type II diabetes and the results for combination therapy with metformin and suphonylurea were difficult to interpret given the results in the other experimental arms [15, 37]. By necessity, we will often need to rely upon observational studies to inform clinical practice and identify priorities for future RCTs.

\section{Interpreting the results of randomized trials using observational studies, risk stratification and simulations}

Observational studies, in many cases, provide more direct data establishing the effectiveness of therapy in a non-experimental setting. Although confounding can never be entirely eliminated, and biases must be taken into account when interpreting observational studies, well-done analyses can illuminate the effects of actual clinical care more directly than RCTs. For example, the Type II diabetes Patient and Outcomes Research Team (PORT) included an evaluation of the effectiveness of starting insulin therapy in standard clinical practice [3]. Randomized controlled trials established that insulin therapy could, under ideal circumstances, result in tight control [4, 38, 39]. This study, conducted in the observational setting, examined, however, the use of insulin therapy in actual clinical practice. The study found, not surprisingly, that starting insulin therapy in real practice was much less effective than in randomized trials and 
that there was no evidence that some physicians were more effective than others in achieving tight control. This study highlights the importance of the gap between efficacy and effectiveness. In clinical research, efficacy is usually defined as the impact of an intervention in the controlled setting of an experimental study, whereas effectiveness is typically defined as the impact of an intervention in usual practice. Unfortunately, as shown in the PORT study, "evidencebased" treatment from randomized trials might not be realized in the absence of the same intensive resources and motivated patients found in RCTs. This has particular ramifications when considering the cost-effectiveness of an intervention; estimates based on RCT data might appear substantially better than they would be in actual clinical practice. Although the Diabetes PORT should not be interpreted as a direct comparison between types of therapy (as in the UKPDS), it did show that intensive glycaemic control will be difficult to achieve in the general population because current management strategies do not typically reach target glycaemic levels that have been seen in resource-intensive RCTs.

In addition to the difficulty of extrapolating from efficacy to effectiveness, applying the average results of RCTs to the individual patients, or even the general population, is problematic. Thus, treatments that are highly effective on average might be mistakenly given to patients who have very little likelihood of benefit and to others who are likely to be harmed. It has been suggested that observational data can be useful to frame the group being treated and to assess whether variations in ethnicity, social factors or comorbid illnesses could change the benefit of a therapy or to attempt to define the baseline risk of the patient, and thus the likely benefit of the treatment [11, 12, 28, 29]. For example, it has been shown using data from the European Carotid Surgery Trial and an independently derived model, that patients with high-grade symptomatic carotid stenosis without other stroke risk factors (i.e. "low-risk" patients) could be more likely to be harmed by surgery than helped, despite the overall benefit found in the trial [40]. The risk of patients was defined by the presence or absence of fifteen clinical variables, such as age, sex, hypertension, diabetes and peripheral vascular disease. Standard sub-group analysis, which considers risk/benefit factors one at a time and ignores that patients have multiple attributes that impact risk and response to therapy simultaneously [31-35] would have been unable to discover that many patients in the study received little or no benefit and that some were being harmed.

In the same way, treatments that are effective on average, could be less effective in many patients to whom they are applied. For example, in Type II diabetes, tight control on average will reduce the probability of microvascular complication [14]. Many pa- tients with Type II diabetes will, however, receive much less than the average benefit seen in randomized controlled trials. For example, the returns from improving haemoglobin $A_{1 c}$ by $2 \%$, as seen in the DCCT, are much greater for patients who start at $11 \%$ than those who start at $8 \%$ [41]. In addition, the effect on the intermediate (early retinopathy and nephropathy) outcomes measured in most clinical trials will not translate into the same level of impact on end-stage outcomes [6, 7]. As discussed above, results from both experimental and observational studies and simulation models [42] must be used to interpret the DCCT and UKPDS. These models show that younger onset patients are likely to receive substantial benefit from tight glycaemic control. These same models show, however, that for older patients with reasonable control on a conventional regimen, the baseline risk of end-stage microvascular complications is so low that very little benefit will be gained from tight glycaemic control. Thus, through use of a combination of simulation modelling and risk stratification based upon RCTs and observational data, treatment can be targeted to high-risk patients and patients at low risk can avoid the costs, inconvenience and complications of intensive therapy. Unfortunately, simulation modelling is limited by the available data and should be interpreted with caution, particularly when RCTs have not shown a causal relation between treatment and outcome. Furthermore, the biases inherent in observational studies are difficult to overcome. Although careful analysis can reduce bias, there are to date only limited examples of studies showing that the predictions from observationally derived models are accurate when compared with the results of randomized studies $[6,43]$. It is of great importance that further studies be carried out.

These methods can further evaluate other options; for example, it is possible to determine the costs andeffects of less frequent retinal screening in low-risk patients with Type II diabetes, such as patients with near-normal glycaemic control, where risk and therefore benefit, is expected to be small. There are growing examples of these techniques in the medical literature. Underlying risk, or severity of disease, has been shown to influence the effectiveness of beta blocking agents in acute myocardial infarction [44] and coronary artery bypass grafting for coronary artery disease [45]. Thrombolytic therapy in acute myocardial infarction is another apt example; one report [46] showed that if patients are risk stratified, most of the incremental benefit of tissue plasminogen activator therapy accrues to a sub-group of high-risk patients. Treatment of low-risk patients, in this instance, can lead not only to little or no benefit but can actually cause harm. Efficacious treatments applied to patients who are unlikely to get much benefit has been termed "flat-of-the-curve medicine" [47], and such 
treatment is felt to account for a large proportion of health care expenditures. With the perpetual discovery of new diagnostic and therapeutic technologies continually driving up the cost of health care, identifying the "flat of the curve" applications of these technologies is increasingly critical to socially responsible medical practice.

Finally, we must do a better job of reporting the results of RCTs. Some authors [48] have proposed a method for reporting risk-stratified results. They suggest that a post-hoc risk score should be assigned to all trial patients based on a logistic-regression model that predicts the probability of the measured outcome based upon known predictors of risk. The trial cohort can then be divided into quartiles based on their expected risk. This method would allow for an evaluation of the degree of variation of risk in the trial cohort and also for comparing whether the absolute and relative risk reduction of the treatment varies for those who have attributes placing them at different levels of baseline risk. Reporting results in this manner will be much more useful than the traditional serial bivariate sub-group analyses (i.e. stratification by age, sex, or individual comorbidities), and should help to better inform clinical practice and subsequent cost-effectiveness and simulation analyses.

\section{Summary}

Randomized trials are often considered the standard for defining the practice of evidence based medicine. Although we wholeheartedly support the need for RCTs to establish causality, the use of observational studies as a lens to re-evaluate the results of randomized trials can help optimize delivery of care, inform clinical practice and determine the need for further RCTs. Using the full richness of the available experimental and observational evidence, combined with better risk-stratification and simulation techniques, can help us provide better and more cost-effective care to our patients. It is essential that we do not rely on the mean effect suggested by experimental data to define the standard of care in patients with diabetes; without use of the full spectrum of epidemiological techniques, we run the risk of treating low-risk patients and providing inefficient or even harmful care.

Acknowledgements. Dr. Kent is a Robert Wood Johnson Clinical Scholar.

\section{References}

1. Doll R (1994) Summation of conference. Doing more good than harm: the evaluation of health care interventions. Ann NY Acad Sci 703:313

2. Javitt JC, Aiello L (1997) Cost-effectiveness of detecting and treating diabetic retinopathy. Ann Intern Med 124: 164-169
3. Hayward RA, Manning WG, Kaplan SH, Wagner EH, Greenfield S (1997) Starting insulin therapy in patients with Type II diabetes: effectiveness, complications, and resource utilization. JAMA 278: 1663-1669

4. The Diabetes Control and Complications Trial Research Group (1993) The Effect of Intensive Treatment of Diabetes on the Development and Progression of Long-Term Complications in Insulin-Dependent Diabetes Mellitus. N Eng J Med 329: 977-986

5. UK Prospective Diabetes Study Group (1998) Cost effectiveness analysis of improved blood pressure control in hypertensive patients with type 2 diabetes: UKPDS 40. BMJ 317: 720-726

6. Vijan S, Hofer TP, Hayward RA (1997) Estimated Benefits of Glycaemic Control in Microvascular Complications in Type II Diabetes. Ann Intern Med 127: 788-795

7. Eastman RC, Javitt JC, Herman WH et al. (1997) Model of complications of NIDDM. II. Analysis of the health benefits and cost-effectiveness of treating NIDDM with the goal of normoglycemia [see comments]. Diabetes Care 20: 735-744

8. Eastman RC, Javitt JC, Herman WH et al. (1997) Model of complications of NIDDM. I. Model construction and assumptions [see comments]. Diabetes Care 20: 725-734

9. Black N (1996) Why we need observational studies to evaluate the effectiveness of health care. Lancet 312: 1215-1218

10. Mant D (1999) Evidence and primary care: Can randomised trials inform clinical decisions about individual patients? Lancet 353: 743-746

11. Glasziou P, Irwig L (1995) An evidence based approach to individualising treatment. BMJ 311: 1356-1359

12. Fahey T (1998) Applying the results of clinical trials to patients to general practice: perceived problems, strengths, assumptions, and challenges for the future. Br J Gen Pract 48: $1173-1178$

13. American Diabetes Association (1998) Economic consequences of diabetes mellitus in the US in 1997. Diabetes Care 21: 296-309

14. UK Prospective Diabetes Study (UKPDS) Group (1998) Intensive blood-glucose control with sulphonylureas or insulin compared with conventional treatment and risk of complications in patients with Type II diabetes (UKPDS 33). Lancet 352: 837-853

15. UK Prospective Diabetes Study Group (1998) Effect of intensive blood-glucose control with metformin on complications in overweight patients with Type II diabetes (UKPDS 34). Lancet 352: $854-865$

16. UK Prospective Diabetes Study Group (1998) Efficacy of atenolol and captopril in reducing risk of macrovascular and microvascular complications in Type II diabetes: UKPDS 39. BMJ 317: 713-720

17. UK Prospective Diabetes Study Group (1998) Tight blood pressure control and risk of macrovascular and microvascular complications in Type II diabetes: UKPDS 38. BMJ 317: 703-713

18. American Diabetes Association (1999) Implications of the United Kingdom Prospective Diabetes Study. Diabetes Care 22: [Suppl] S27-S31

19. Hulley S, Cummings S (eds) (1988) Designing Clinical Research. 1st edn. Williams \& Wilkins Baltimore

20. Pirart J (1978) Diabetes Mellitus and Its Degenerative Complications: A Prospective Study of 4400 Patients Observed Between 1947 and 1973. Diabetes Care 1: 168-188, 252-261

21. Klein R, Klein BEK, Moss SE et al. (1988) Glycosylated hemoglobin predicts the incidence and progression of diabetic retinopathy. JAMA 260: 2864-2871 
22. Omenn GS (1998) Chemoprevention of lung cancer: the rise and demise of beta-carotene. Annu Rev Public Health 19: 73-99

23. Summary of the Second Report of the National Cholesterol Program (NCEP) Expert Panel on Detection, Evaluation, and Treatment of High Blood Cholesterol in Adults (Adult Treatment Panel II). (1990) JAMA 264: 723-726

24. Ward LC, Fielding JWL, Dunn JA, Kelly KA (1992) The selection of cases for randomized trials: a registry of concurrent trial and non-trial participants. Br J Cancerl 66: 943-962

25. Stiller CA (1994) Decentralised treatment, entry to trials, and survival. Br J Cancer 70: 352-362

26. Cross design synthesis: a new strategy for studying medical outcomes? (1992) [editorial] Lancet 340: 944-946

27. Stampfer M (1997) Observational epidemiology is the preferred means of evaluating effects of behavioral and lifestyle modification. Controll Clin Trials 18: 494-499

28. Dans AL, Dans LF, Guyatt GH, Richardson S (1998) Users' guides to the medical literature: XIV. How to decide on the applicability of clinical trial results to your patient. Evidence-Based Medicine Working Group. JAMA 279: 545-549

29. Guyatt GH, Sackett DL, Cook DJ (1994) Users' guides to the medical literature. II. How to use an article about therapy or prevention. B. What were the results and will they help me in caring for my patients? Evidence-Based Medicine Working Group. JAMA 271: 59-63

30. Ioannidis J, Lau J (1997) The impact of high-risk patients on the results of clinical trials. J Clin Epidemiol 50: 1089-1098

31. Horwitz RI (1996) The dark side of evidence-based medicine. Cleve Clin J Med 63: 320-323

32. Buyse ME (1989) Analysis of clinical trial outcomes: some comments on subgroup analyses. Control Clin Trials 10: 187S-194S

33. Detsky AS, Naglie IG (1995) Subgroup analyses: primary and secondary [editorial]. ACP J Club 122:A12-A14

34. Yusuf S, Wittes J, Probstfield J, Tyroler HA (1991) Analysis and interpretation of treatment effects in subgroups of patients in randomized clinical trials. JAMA 266: 93-98

35. Oxman AD, Guyatt GH (1992) A consumer's guide to subgroup analyses. Ann Int Med 116: 78-84
36. Naylor C (1995) Grey zones of clinical practice: Some limits to evidence-based medicine. Lancet 345: 840-842

37. Nathan D (1998) Some answers, more controversy, from UKPDS. Lancet 352 (commentary): 832-834

38. Ohkubo Y, Kishikawa H, Araki E et al. (1995) Intensive insulin therapy prevents the progression of diabetic microvascular complications in Japanese patients with nondependent diabetes mellitus: a randomized prospective 6year study. Diabetes Res Clin Pract 28: 103-117

39. Abraira C, Colwell JA, Nuttall FQ et al. (1995) Veterans Affair Cooperative Study on Glycemic Control and Complications in Type II Diabetes (VA CSDM): Results of the feasibility trial. Diabetes Care 18: 1113-1123

40. Rothwell P (1995) Can overall results of clinical trials be applied to all patients? Lancet 345: 1616-1619

41. The Diabetes Control and Complications Trial Research Group (1995) The Relationship of Glycemic Exposure (HBA 1 c) to the Risk of Development and Progression of Retinopathy in the Diabetes Control and Complications Trial. Diabetes 44: 968-983

42. Sonnenberg FA, Beck JR (1993) Markov models in medical decision making: a practical guide. Med Decis Making 13: $322-338$

43. Hlatky MA, Califf RM, Harrell FE, Lee KL, Mark DB, Pryor D (1988) Comparison of predictions based on observational data with the results of randomised controlled trials of coronary artery bypass surgery. J Am Coll Cardiol 11: 237-245

44. Yusuf S, Peto R, Lewis J, Collins R, Sleight P (1985) Beta blockade during and after myocardial infarction. Prog Cardiovasc Dis 27: 335-371

45. Califf R, Harrel F, Lee K et a. (1989) The evolution of medical and surgical therapy for coronary artery disease. A 15 year perspective. JAMA 261: 2077-2086

46. Califf R, Woodlief L, Harrell F et al. Selection of thrombolytic therapy for individual patients: Development of a clinical model. Am Heart J 133: 630-639

47. Fuchs V (1972) Healthcare and the United States economic system: An essay in abnormal physiology. Millbank Q 50: 211-237

48. Ioannidis J, Lau J (1998) Heterogeneity of the baseline risk within patient populations of clinical trials: A proposed evaluation algorithm. Am J Epidemiol 148: 117-1126 